\title{
The application of genetics approaches to the study of exceptional longevity in humans: potential and limitations
}

\author{
Anna Ferrario ${ }^{1}$, Francesco Villa ${ }^{1}$, Alberto Malovini ${ }^{2}$, Fiorella Araniti ${ }^{1}$ and Annibale A Puca ${ }^{1,3^{*}}$
}

\begin{abstract}
The average life-span of the population of industrialized countries has improved enormously over the last decades. Despite evidence pointing to the role of food intake in modulating life-span, exceptional longevity is still considered primarily an inheritable trait, as pointed out by the description of families with centenarian clusters and by the elevated relative probability of siblings of centenarians to become centenarians themselves. However, rather than being two separate concepts, the genetic origin of exceptional longevity and the more recently observed environment-driven increase in the average age of the population could possibly be explained by the same genetic variants and environmentally modulated mechanisms (caloric restriction, specific nutrients). In support of this hypothesis, polymorphisms selected for in the centenarian population as a consequence of demographic pressure have been found to modulate cellular signals controlled also by caloric restriction. Here, we give an overview of the recent findings in the field of the genetics of human exceptional longevity, of how some of the identified polymorphisms modulate signals also influenced by food intake and caloric restriction, of what in our view have been the limitations of the approaches used over the past years to study genetics (sib-pair-, candidate gene association-, and genome-wide association-studies), and briefly of the limitations and the potential of the new, high-throughput, next-generation sequencing techniques applied to exceptional longevity.
\end{abstract}

Keywords: Aging, Centenarians, Longevity

\section{Mechanisms of longevity}

Life expectancy in the US 1900 Birth Cohort Study was found to be 51.5 years for males and 58.3 years for females, and currently $1 / 10,000$ individuals reach 100 years of age: this prevalence is quickly changing and will probably soon approach 1/5,000 [1]. The increased ability to reach 100 years old in industrialized countries over the last 160 years most likely reflects a rise in life expectancy - quantified as 3 months/year for females as a consequence of improvements in diet and a reduced exposure to infection and inflammation [2]. In favour of diet as a modulator of longevity, the Elderly Prospective Cohort Study (EPIC) identified a reduced overall mortality among the elderly consuming a modified Mediterranean

\footnotetext{
*Correspondence: puca@longevita.org

${ }^{1}$ IRCCS Multimedica, Via Fantoli 16/15, 20138 Milan, Italy

${ }^{3}$ Università degli Studi di Salerno, Via S. Allende, 84081 Baronissi, Italy

Full list of author information is available at the end of the article
}

diet in which saturated fatty acids were substituted for monounsaturated ones [3].

Centenarians, despite being exposed to the same environmental conditions as members of the average population, manage to live much longer; moreover, as a consequence of demographic selection, centenarians have a compression of morbidity and mortality towards the end of their life-span [4]. Genetically, this compression in morbidity and mortality is correlated with the enrichment of protective alleles and the depletion of detrimental ones. These alleles run in families, as shown by the familiar clustering of exceptional longevity. It has been estimated that genetic variants account for at least $25 \%$ of human life-span, and for even a larger proportion in individuals living to extreme age $[5,6]$.

The potential overlap of hits for environmentally and genetically mediated predisposition for extreme longevity in centenarians is highlighted by the association of genetic variants of genes that regulate, or that are 
regulated by, nutrient metabolism, such as apolipoprotein E (APOE) and Forkhead box O3A (FOXO3A) [7]. Inheritance of the longevity phenotype is underlined by the low all-cause and cardiovascular-disease mortality rates observed in offspring of centenarians when compared with an age-matched population [8]. The study of centenarian offspring has revealed biomarkers of longevity, like low serum levels of heat shock proteins (HSP), large lipid particle sizes, and high membrane palmitoleic acid paired with a low peroxidation index [9-11]. In addition, centenarians have high glucose tolerance and insulin action, and low heart-rate variability (HRV), which contrasts with the decline observed in control populations [12,13].

Genetic variants that modulate human longevity should also modulate cellular pathways that control key aspects of the aging process, such as oxidative stressinduced apoptosis (RAS/ERK pathway), DNA repair (NF-KB1 and hTERT), senescence (p53), mitochondrial biogenesis (AMPK) and cell survival (PI3K/AKT pathway). Many of these pathways cross-talk with each other and are finely regulated in order to obtain the best tradeoff between advantages and disadvantages of inducing either cell survival or apoptosis/senescence. The best trade-off is mostly tissue specific and can change with the degree of the tissue's differentiation (stem versus differentiated cells) and health status (healthy, proliferative, degenerative or ischemic). It is plausible that genetic variants that impact on human longevity affect genes that are expressed selectively in some tissues and/or in specific stages of differentiation. Although aging has been considered an evolutionary adaptation for fighting cancer through the activation of processes like senescence, there are signals that are able to activate cell-specific responses, e.g. induction of apoptosis by adenosine monophosphateactivated protein kinase (AMPK) in cancer cells, while inducing survival in healthy cells. AMPK recognizes high levels of AMP, and is activated by caloric restriction, physical exercise, metformin, essential amino acids and alpha-lipoic acid [14]. Furthermore, AMPK induces mitochondrial biogenesis, autophagy and beta-oxidation of free fatty acids [15-17]. Reduction of fatty acid betaoxidation promotes diabetes, obesity and, ultimately, aging.

How can these cellular signals be altered without producing side effects? The answer could be found in the centenarian genome through the identification of genetic variants that have been selected or dropped for their role in human health. AMPK signalling is an example of how variation in the quality and the amount of food could impact on longevity by modulating signals that are influenced by genetic variants selected in centenarians. To be noted, Sirtuin 1 (SIRT1), despite its role in many cellular processes, induces survival and is not affected by polymorphisms that associate with exceptional longevity in humans, perhaps on account of its critical role in tumours as well [18].

\section{The candidate gene approach and associations with longevity}

To date, only a few genetic variants have been consistently found associated with exceptional longevity in humans (Table 1). The most convincing result discovered to date by a candidate gene approach is the reduction of the APOE 4 allele in centenarians as a result of its correlation with cardiovascular disease and Alzheimer's disease [7]. APOE knockout mice develop atherosclerosis, so genes that modulate vascular integrity are potential candidates that may be identified in genetic association studies of exceptional longevity [19].

The FOXO3A rs2802292 allele is another variant found associated with exceptional longevity across populations [20,22-24]. Nevertheless, this polymorphism has no apparent impact on the functions of FOXO3A and it is not in LD with functional variants. However, FOXO3A is part of a pathway associated with longevity: IGF1/ $\mathrm{PI} 3 \mathrm{~K} / \mathrm{PDK} 1 / \mathrm{AKT} / \mathrm{FOXO}$. Animal studies from worms to mice have shown that genetic modifications are able to postpone aging by modulating the IGF1/PI3K/PDK1/ AKT/FOXO pathway [25]. This pathway regulates many aspects of cell homeostasis, from cell survival and proliferation to oxidative stress response, depending on concomitant stimuli [26,27]. Interestingly, individuals with short stature due to a lack of growth hormone, which is upstream of the IGF1/PI3K/PDK1/AKT/FOXO pathway, have a reduced incidence of tumours and diabetes [28]. The IGF1/PI3K/PDK1/AKT/FOXO pathway is strongly modulated by caloric restriction, as are the levels of AMPK and SIRT1. Glucose and insulin resistance also modulates this pathway, which associates with inefficient uptake of glucose, inducing its overstimulation and aging. To some extent, genetic alterations of the insulin-like growth factor 1 (IGF1) receptor that alter the IGF signalling pathway confer an increase in propensity for longevity [29].

Adenosine deaminase, RNA-specific (ADAR) and telomerase gene variants have also been associated with human longevity [30,31]. However, with the exception of APOE and FOXO3A variants, none of the many candidate genetic variants tested to date have been consistently replicated across populations. This is possibly on account of differing environmental stimuli generating inconsistent demographic pressures, making results, as a consequence, irreproducible [32].

Other potential problems that generate false positive and false negative results include the low power of studies using small sample sizes, and a lack of a suitable control for the genetic admixture. On the first point, an 
Table 1 Genes and variants found correlated with longevity in humans

\begin{tabular}{llllll}
\hline Gene & Variant & Occurrence in centenarians & Previous disease correlations & Potential role in longevity & Ref. \\
\hline APOE & $\varepsilon 4^{*}$ & reduced & Alzheimer's and cardiovascular diseases & Maintenance of vascular integrity & [7] \\
FOXO3A & rs2802292* & more present & none & Control of cell homeostasis \\
CAMK4 & rs $10491334^{\dagger}$ & more present & hypertension & Modulation of CREB, SIRT and FOXO3A & [21] \\
ATXN1 & rs697739 & more present & amyotrophic lateral sclerosis (age of onset) & Modulation of CREB & [21] \\
DCAMKL1 & rs9315385 & more present & heart rate variability & Modulation of CREB & [21] \\
\hline
\end{tabular}

${ }^{*}$, result obtained by a candidate gene approach and replicated in other studies; ${ }^{\dagger}$, result obtained from a GWAS and confirmed in a replication cohort.

extremely instructive review has been written by Altshuler, Daly and Lender, who calculate the power of a study based on the number of individuals genotyped, the number of tested hypotheses, and the frequency of the allele tested for a specific OR [33]. From the graph given in their review (Figure 1), it is clear that for the OR expected in human exceptional longevity (between 1.2 and 2), the power of a study is highly dependent upon the number of hypotheses tested. If we consider that many laboratories test their genetic variants and publish only the positive results they find, the few hundred individuals typically used in a candidate gene approach on exceptional longevity are not sufficient to minimize false-positive findings.

With regard to the appropriate control for a given genetic admixture, it is possible to correct for systematic ancestry differences between cases and controls - an effect that can cause false associations - by the application of principal components analysis to the genotyping of thousands of SNPs with chips [34].

Finally, because the candidate gene approach is hypothesis driven, the functional validation of the coded protein in signals important for aging and longevity do not add strength to the finding, while as we will see, this is an opportunity for hypothesis-free approaches, such as the genome-wide association study (GWAS) and sib-pair analysis.

\section{Genome-wide association studies and exceptional longevity}

GWASs are hypothesis-free efforts that generate findings that need to be replicated in independent populations. In the case of exceptional longevity, success in replicating initial findings is negatively influenced by the differences in participant ages, gender and disease status distribution across the analysed populations. Furthermore, the

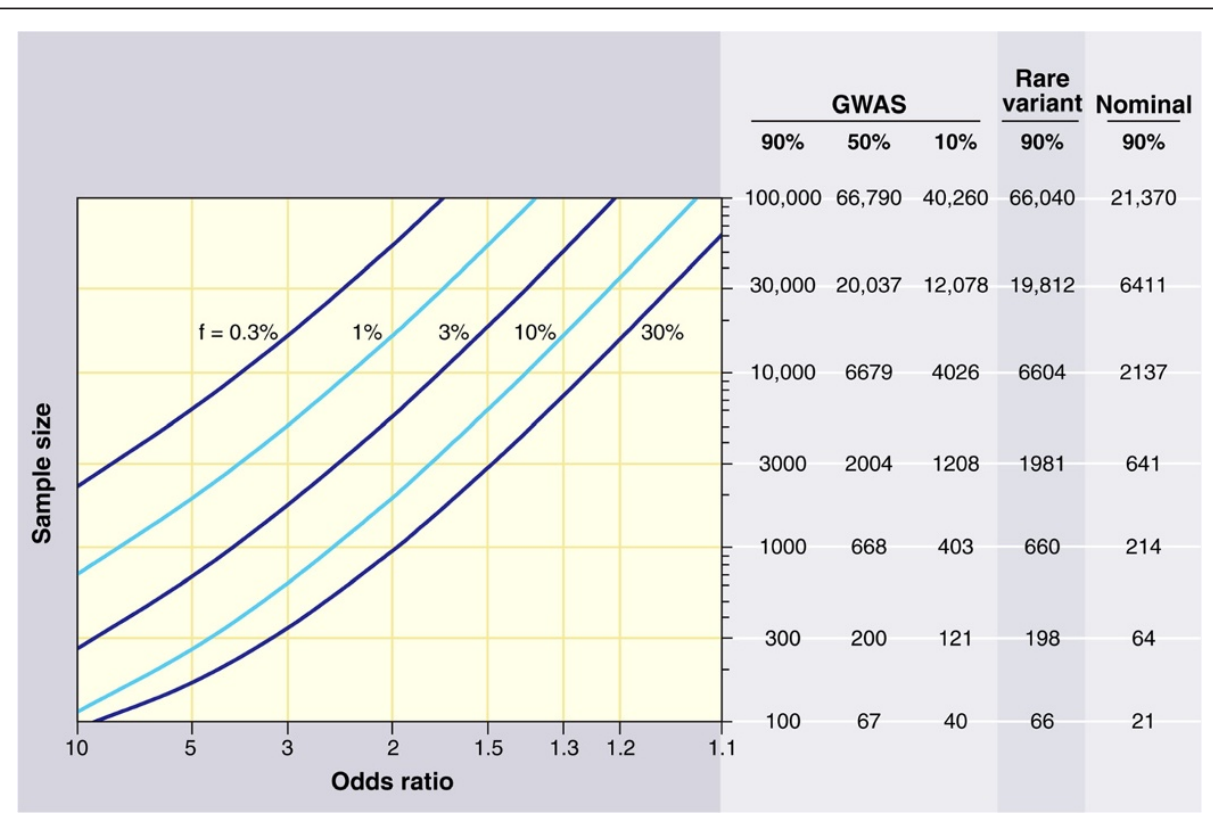

Figure 1 Sample sizes required for genetic association studies. The graph shows the total number $\mathrm{N}$ of samples (consisting of N/2 cases and $N / 2$ controls) required to map a genetic variant as a function of the increased risk due to the disease-causing allele ( $x$ axis) and the frequency of the disease-causing allele (various curves). The required sample size is shown in the table on the right for various different kinds of association studies [33]. Reproduced with permission from The American Association for the Advancement of Science. 
GWAS approach suffers from the multiple-testing statistical penalty that forces the adoption of very low $p$ values of significance, hence favouring the phenomenon of the winning course, i.e. the enrichment of false-positive associations among the dozens of top findings [35]. For these reasons, recent GWASs have failed to find variants that cross-validate across populations - with the exception of the known APOE locus - pointing to the need of much larger studies or alternative study designs in order to discover common polymorphisms with smaller genetic effects and rare variants with high penetrance that influence exceptional longevity [36,37].

Regarding the power of capturing true associations in GWAS efforts, the Altshuler, Lander and Daly review calculation clearly shows that a sample size comprising thousands of individuals is needed to identify the expected OR in a GWAS on exceptional longevity [33]. Thus, a GWAS on exceptional longevity can be considered only a hypothesis-generating effort to be used in conjunction with other studies.

For the above reasons, in our recently published GWAS on individuals enrolled in the Southern Italian Centenarian Study (SICS), we not only attempted to progressively reduce the number of tested hypotheses, but also considering the redundancy (non-independence) of the many SNPs represented on the Illumina $317 \mathrm{k}$ beadchip employed, we decided to use a $300 \mathrm{k}$ SNP screening of the SICS individuals as a hypothesis-generating set, adopting a Genomic Control (GC)-corrected p-value $<1 \mathrm{e}-4$ threshold (which is a less stringent threshold than $p<5 \times 10-2 / 317000=1.5 \times 10-7)$ for the replication, evaluating allelic, genotypic, dominant and recessive genetic association models [21].

Initial screening of SICS individuals identified CAMK4 rs10491334, a variant that had been already established among the top 5 SNPs in the Framingham Heart Study on diastolic high blood pressure [38]. The fact that CAMK4 rs10491334 associates also with hypertension is reassuring in that hypertension and longevity are regulated by common pathways. In fact, mice with genetic ablation of the angiotensin II type1 receptor - the key regulator of blood pressure - had increased expression of the longevity gene Sirt3 and improved survival [39]. Interestingly, rs10491334 correlated with CAMK4 protein expression, and functional studies revealed the ability of CAMK4 protein to modulate SIRT1 and FOXO3A.

The ataxin-1 (ATXN1) rs697739 allele was another variant found among the top findings of our GWAS on SICS individuals. This polymorphism had been previously associated with the age of onset of sporadic amyotrophic lateral sclerosis, a disease of unknown cause characterized by slowly progressive degeneration of motor neurons and that usually occurs in patients aged 40-60 years [40]. ATXN1 is the gene responsible for spinocerebellar ataxia type 1 and antagonizes the neuronal survival function of myocyte enhancer factor-2 (MEF2) [41].

MEF2 transcription repression by cabin1-HDAC4 is removed by CAMKIV activation, and this suggests that MEF2 is a common downstream target of CAMKIV and ATXN1 $[42,43]$.

In addition to CAMKIV rs10491334 and ATXN1 rs697739, the rs9315385 allele of doublecortin and $\mathrm{Ca}^{2+} /$ calmodulin-dependent kinase-like-1 (DCAMKL1) was a third top finding of our study. DCAMKL1 has structural similarity with CAMKIV, but despite this, it represses CAMKIV-induced activation of cAMP response element-binding (CREB) protein via phosphorylation of transducer of regulated CREB activity 2 (TORC2) at Ser171 [44]. DCAMKL1 rs9315385 was previously associated with total power of HRV [45]. A reduced HRV is a marker of autonomic dysfunction and is associated with an increased risk of cardiovascular morbidity and mortality [46]. HRV-parasympathetic function decreases up to the eighth decade of life, followed by an increase to higher levels - similar to those found in a younger population - in nonagenarians and centenarians [13]. Similarly to CAMKIV, DCAMKL1 and ATXN1 are expressed mainly in brain. These data support the importance of the CAMKIV/CREB pathway in regulating the aging process.

A brief mention needs to be made here on the cuttingedge, genetic signature paper by Sebastiani et al. that very elegantly proved that a complex analysis on 281 SNPs allowed to define clusters of individuals that aged differently based on their genetic signature [47].

\section{Linkage analysis and exome sequencing}

Sib-pair analysis has been for a while the only tool available for the identification of chromosomal regions that potentially harbour genetic variants influencing the phenotype of interest. The approach can identify excess allele sharing, and was initially performed with microsatellites. It consists in the identical-by-descendant analysis of very informative markers that reconstruct the haplotype of parents and how they co-segregate in their offspring. We performed such an analysis on a unique collection of sib-pairs and their families, collected by the New England Centenarian Study (NECS), and identified a significant peak on 4q25 [48]. Follow-up analysis failed to identify genetic variants that could explain the initial linkage finding. Rare mutations that segregate in centenarian siblings are eventually captured by sibling-pair analysis, but this cannot be the case for genetic association studies that loose power as the allele frequency of the tested polymorphisms drops. Furthermore, it is possible that linkage efforts identify chromosomal regions where more causative genetic variants reside, and thus the sum of their effects determines the linkage result, whereas 
with follow-up genetic association approaches, the analysis involves one common polymorphism at a time or, eventually, haplotypes. Attempts to replicate the initial linkage did not succeed, except for an initial replication effort that successfully replicated the linkage at D4S1564 $[49,50]$. A negative replication effort can be due to an initial false-positive finding or to the diversity of the populations used for the replication effort, in terms of genetic background, the environment applying the demographic pressure, the ages of the participants, the number of families and the genetic markers adopted. Recently, Kunkel's laboratory published a well-performed re-analysis of a part of the sib-pairs used in the initial study, plus new sib-pairs recruited by Elixir Pharmaceuticals [51]. To be noted, some of the largest and more impressive families - those that were genotyped upfront and that showed immediately a significant linkage on $4 q 25$ in the original study - were either not analysed or done so only in part by this second effort. The new analysis adopted a high-density marker panel of SNPs to genotype the patients, allowing a better coverage of the genome. They did not replicate the chromosome $4 \mathrm{q} 25$ finding, except when the same stringent criteria were adopted to select a sub-set of centenarian families. Interestingly, a new peak on chromosome 3p24-22 reached significant threshold, and a second peak was highly suggestive of linkage at 9q31-34. This latter peak appeared also in the previous analysis with microsatellites, even if less robustly. The attempt to identify the genetic variant/variants responsible for the $4 \mathrm{q} 25$ peak pointed to the initial encouraging genetic variant in the promoter of the microsomal triglyceride transfer protein (MTP) gene [52]. Unfortunately, the finding was not replicated by an independent effort and by our analysis that included more controls [32,53].

It is plausible that different approaches are needed to follow up genetic linkage findings, to point to the identification of rare variants that co-segregate in families. To this end, exome sequencing data, intersected with linkage data, could give rise to interesting results.

To be noted, the 4q25 locus harbours elongation of very long chain fatty acids protein 6 (ELOVL6), the elongase that transforms $\mathrm{C} 16: 0$ into $\mathrm{C} 18: 0$ and $\mathrm{C} 16: 1$ into C18:1. Polymorphisms in this gene have been associated with insulin sensitivity; a mouse deficient for this gene carried high doses of C16:1 (palmitoleic acid) and did not acquire insulin resistance after a high-fat diet $[54,55]$. C16:1 has been identified as an adipose tissuederived lipid hormone that strongly stimulates muscle insulin action and suppresses hepatosteatosis [56]. Genetically modified, long-living worms have an incredible correlation between their increase in life-span and their palmitoleic acids levels [57]. This is stunning if we consider the increased level of palmitoleic acid that we observed in centenarians' offspring and that the gene of the major modifier of palmitoleic acid levels (i.e. ELOVL6) is located in the 4q25 longevity locus [11,48]. The re-sequencing in centenarians of this gene could bring to the identification of rare variants able to influence its activity.

Thus, the old approach of linkage analysis when combined with the new technologies of high massive resequencing could produce novel and interpretable results. Re-sequencing alone, because of the enormous amount of information generated, would force the application of a huge statistical correction for the multiple testing, which would cause the loss of most, if not all, the potential findings, as happens with GWAS.

Furthermore, multivariate models, based on machinelearning algorithms (i.e. Bayesian networks [58], classification and regression trees - CART [59] - and support vector machines - SVM [60]), are able to overcome the limitations of the usual "one-SNP-at-the-time" testing strategies usually employed for identifying causative variants. In particular, these kinds of approaches allow for a more in-depth comprehension of the molecular mechanisms underlying multifactorial traits, such as longevity, which result from the interaction of genetic variants (SNPs, mutations) and environmental and clinical determinants (e.g. diet, stress, comorbidities). In this context, bioinformatics plays a key role, allowing genetic information to be managed at a genome-wide level and to be integrated with the clinical information available.

\section{Concluding remarks}

Despite the enormous progress achieved by DNAinvestigating technologies, such as SNP arrays and exome capturing/re-sequencing, the current knowledge on how genetic variants influence exceptional longevity in humans is still based on the old candidate gene approaches. The adoption of innovative study designs combined with novel genetic platforms and innovative statistical methods hopefully will bring to the identification of new intervention points at which to modulate aging and the diseases of aging.

\footnotetext{
Abbreviations

ADAR: adenosine deaminase RNA-specific; AMPK: Adenosine

monophosphate-activated protein kinase; APOE: apolipoprotein E;

ATXN1: Ataxin 1; CAMK: $\mathrm{Ca}^{2+} /$ calmodulin-dependent protein kinase;

CART: classification and regression tree; CREB: CAMP response element-binding;

DCAMKL1: doublecortin and $\mathrm{Ca}^{2+} /$ calmodulin-dependent kinase-like-1;

ELOVL6: elongation of very long chain fatty acids protein 6; EPIC: Elderly

Prospective Cohort Study; FOXO: forkhead box O; GC: genomic control;

GWAS: genome-wide association study; HRV: heart-rate variability; HSP: heat shock

protein; IGF: insulin-like growth factor; LD: linkage disequilibrium; MEF2: myocyte enhancer factor-2; MTP: microsomal triglyceride transfer protein; NECS: New

England Centenarian Study; OR: odds ratio; SICS: Southern Italian Centenarian

Study; SIRT1: Sirtuin 1; SNP: single nucleotide polymorphism; SVM: support vector

machine; TORC2: transducer of regulated CREB activity 2.
} 


\section{Competing interests}

The authors declare that they have no competing interests.

\section{Acknowledgements}

Anna Ferrario was supported by a Fondazione Umberto Veronesi fellowship. We thank Michael V. G. Latronico for careful revision of the manuscript.

\section{Author details}

${ }^{1}$ IRCCS Multimedica, Via Fantoli 16/15, 20138 Milan, Italy. ${ }^{2}$ University of Pavia, Via Ferrata 1, 27100 Pavia, Italy. ${ }^{3}$ Università degli Studi di Salerno, Via S. Allende, 84081 Baronissi, Italy.

\section{Authors' contributions}

AAP wrote the first draft; Subsequent drafts were written by AF, FV and FA, who had the overall supervision of the review processing; all authors edited the paper and approved its final version.

Received: 27 March 2012 Accepted: 23 April 2012

Published: 23 April 2012

\section{References}

1. Perls TT: The different paths to 100. Am J Clin Nutr 2006, 83:484S-487S.

2. Oeppen J, Vaupel JW: Demography. Broken limits to life expectancy. Science 2002, 296:1029-1031.

3. Trichopoulou A, Orfanos P, Norat T, Bueno-de-Mesquita B, Ocke MC, Peeters $\mathrm{PH}$, van der Schouw YT, Boeing $H$, Hoffmann $\mathrm{K}$, Boffetta $\mathrm{P}$, et al: Modified Mediterranean diet and survival: EPIC-elderly prospective cohort study. BMJ 2005, 330:991.

4. Terry DF, Sebastiani $P$, Andersen SL, Perls TT: Disentangling the roles of disability and morbidity in survival to exceptional old age. Arch Intern Med 2008, 168:277-283.

5. Perls T, Shea-Drinkwater M, Bowen-Flynn J, Ridge SB, Kang S, Joyce E, Daly M, Brewster SJ, Kunkel L, Puca AA: Exceptional familial clustering for extreme longevity in humans. J Am Geriatr Soc 2000, 48:1483-1485.

6. Perls T, Wilmoth J, Levenson R, Drinkwater M, Cohen M, Bogan H, Joyce $E_{i}$ Brewster S, Kunkel L, Puca A: Life-long sustained mortality advantage of siblings of centenarians. Proc Natl Acad Sci U S A 2002, 99:8442-8447.

7. Schachter F, Faure-Delanef L, Guenot F, Rouger H, Froguel P, Lesueur-Ginot L, Cohen D: Genetic associations with human longevity at the APOE and ACE loci. Nat Genet 1994, 6:29-32.

8. Terry DF, Wilcox MA, McCormick MA, Pennington JY, Schoenhofen EA, Andersen SL, Perls TT: Lower all-cause, cardiovascular, and cancer mortality in centenarians' offspring. J Am Geriatr Soc 2004, 52:2074-2076.

9. Terry DF, McCormick M, Andersen S, Pennington J, Schoenhofen E, Palaima E, Bausero M, Ogawa K, Perls T, Asea A: Cardiovascular disease delay in centenarian offspring: role of heat shock proteins. Ann N Y Acad Sci 2004, 1019:502-505

10. Barzilai N, Atzmon G, Schechter C, Schaefer EJ, Cupples AL, Lipton R, Cheng S, Shuldiner AR: Unique lipoprotein phenotype and genotype associated with exceptional longevity. JAMA 2003, 290:2030-2040.

11. Puca AA, Andrew P, Novelli V, Anselmi CV, Somalvico F, Cirillo NA, Chatgilialoglu C, Ferreri C: Fatty acid profile of erythrocyte membranes as possible biomarker of longevity. Rejuvenation Res 2008, 11:63-72.

12. Paolisso G, Gambardella A, Ammendola S, D'Amore A, Balbi V, Varricchio M, D'Onofrio F: Glucose tolerance and insulin action in healty centenarians. Am J Physiol 1996, 270:E890-E894.

13. Zulfiqar U, Jurivich DA, Gao W, Singer DH: Relation of high heart rate variability to healthy longevity. Am J Cardiol 2010, 105:1181-1185.

14. Hardie DG: Adenosine Monophosphate-Activated Protein Kinase: A Central Regulator of Metabolism with Roles in Diabetes, Cancer, and Viral Infection. Cold Spring Harb Symp Quant Biol 2011, [Epub ahead of print].

15. Zong H, Ren JM, Young LH, Pypaert M, Mu J, Birnbaum MJ, Shulman Gl: AMP kinase is required for mitochondrial biogenesis in skeletal muscle in response to chronic energy deprivation. Proc Natl Acad Sci U S A 2002, 99:15983-15987.

16. Hardie DG: AMPK and autophagy get connected. EMBO J 2011, 30:634-635.

17. Hardie DG: AMPK: a key regulator of energy balance in the single cell and the whole organism. Int J Obes (Lond) 2008, 32(Suppl 4):S7-S12.
18. Flachsbart F, Croucher PJ, Nikolaus S, Hampe J, Cordes C, Schreiber S, Nebel A: Sirtuin 1 (SIRT1) sequence variation is not associated with exceptional human longevity. Exp Gerontol 2006, 41:98-102.

19. Imaizumi K: Diet and atherosclerosis in apolipoprotein E-deficient mice. Biosci Biotechnol Biochem 2011, 75:1023-1035.

20. Willcox BJ, Donlon TA, He Q, Chen R, Grove JS, Yano K, Masaki KH, Willcox DC, Rodriguez B, Curb JD: FOXO3A genotype is strongly associated with human longevity. Proc Natl Acad Sci U S A 2008, 105:13987-13992.

21. Malovini A, Illario M, laccarino G, Villa F, Ferrario A, Roncarati R, Anselmi CV,

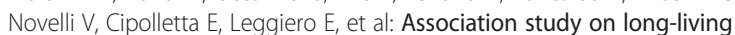
individuals from Southern Italy identifies rs10491334 in the CAMKIV gene that regulates survival proteins. Rejuvenation Res 2011, 14:283-291.

22. Anselmi CV, Malovini A, Roncarati R, Novelli V, Villa F, Condorelli G, Bellazzi R, Puca AA: Association of the FOXO3A locus with extreme longevity in a southern Italian centenarian study. Rejuvenation Res 2009, 12:95-104.

23. Flachsbart $F$, Caliebe $A$, Kleindorp $R$, Blanche $H$, von Eller-Eberstein $H$ Nikolaus S, Schreiber S, Nebel A: Association of FOXO3A variation with human longevity confirmed in German centenarians. Proc Natl Acad Sci U S A 2009, 106:2700-2705.

24. Soerensen M, Dato S, Christensen K, McGue M, Stevnsner T, Bohr VA, Christiansen L: Replication of an association of variation in the FOXO3A gene with human longevity using both case-control and longitudinal data. Aging Cell 2010, 9:1010-1017.

25. Kenyon CJ: The genetics of ageing. Nature 2010, 464:504-512.

26. Kops GJ, Dansen TB, Polderman PE, Saarloos I, Wirtz KW, Coffer PJ, Huang $\Pi$, Bos $J$, Medema RH, Burgering BM: Forkhead transcription factor FOXO3a protects quiescent cells from oxidative stress. Nature 2002, 419:316-321.

27. van der Horst A, Burgering BM: Stressing the role of FoxO proteins in lifespan and disease. Nat Rev Mol Cell Biol 2007, 8:440-450.

28. Guevara-Aguirre J, Balasubramanian P, Guevara-Aguirre M, Wei M, Madia F, Cheng CW, Hwang D, Martin-Montalvo A, Saavedra J, Ingles S, et al: Growth hormone receptor deficiency is associated with a major reduction in pro-aging signaling, cancer, and diabetes in humans. Sci Trans/ Med 2011, 3:70ra13.

29. Pawlikowska L, Hu D, Huntsman S, Sung A, Chu C, Chen J, Joyner AH, Schork NJ, Hsueh WC, Reiner AP, et al: Association of common genetic variation in the insulin/IGF1 signaling pathway with human longevity. Aging Cell 2009, 8:460-472.

30. Sebastiani P, Montano M, Puca A, Solovieff N, Kojima T, Wang MC, Melista E, Meltzer M, Fischer SE, Andersen S, et al: RNA editing genes associated with extreme old age in humans and with lifespan in C. elegans. PLoS One 2009, 4:e8210

31. Atzmon G, Cho M, Cawthon RM, Budagov T, Katz M, Yang X, Siegel G, Bergman A, Huffman DM, Schechter CB, et al: Evolution in health and medicine Sackler colloquium: Genetic variation in human telomerase is associated with telomere length in Ashkenazi centenarians. Proc Natl Acad Sci U S A 2010, 107 (Suppl 1):1710-1717.

32. Novelli V, Viviani Anselmi C, Roncarati R, Guffanti G, Malovini A, Piluso G, Puca AA: Lack of replication of genetic associations with human longevity. Biogerontology 2008, 9:85-92.

33. Altshuler D, Daly MJ, Lander ES: Genetic mapping in human disease. Science 2008, 322:881-888.

34. Price AL, Patterson NJ, Plenge RM, Weinblatt ME, Shadick NA, Reich D: Principal components analysis corrects for stratification in genome-wide association studies. Nat Genet 2006, 38:904-909.

35. loannidis JP, Thomas G, Daly MJ: Validating, augmenting and refining genome-wide association signals. Nat Rev Genet 2009, 10:318-329.

36. Deelen J, Beekman M, Uh HW, Helmer Q, Kuningas M, Christiansen L, Kremer D, van der Breggen R, Suchiman HE, Lakenberg N, et al: Genome-wide association study identifies a single major locus contributing to survival into old age; the APOE locus revisited. Aging Cell 2011, 10:686-698.

37. Nebel $A$, Kleindorp $R$, Caliebe $A$, Nothnagel M, Blanche $H$, Junge $O$, Wittig $M$ Ellinghaus D, Flachsbart F, Wichmann HE, et al: A genome-wide association study confirms APOE as the major gene influencing survival in long-lived individuals. Mech Ageing Dev 2011, 132:324-330.

38. Levy D, Larson MG, Benjamin EJ, Newton-Cheh C, Wang TJ, Hwang SJ, Vasan RS, Mitchell GF: Framingham Heart Study 100 K Project: genome-wide associations for blood pressure and arterial stiffness. BMC Med Genet 2007, 8(Suppl 1):S3. 
39. Benigni A, Corna D, Zoja C, Sonzogni A, Latini R, Salio M, Conti S, Rottoli D, Longaretti L, Cassis P, et al: Disruption of the Ang II type 1 receptor promotes longevity in mice. J Clin Invest 2009, 119:524-530.

40. Landers JE, Melki J, Meininger V, Glass JD, van den Berg LH, van Es MA, Sapp PC, van Vught PW, McKenna-Yasek DM, Blauw HM, et al: Reduced expression of the Kinesin-Associated Protein 3 (KIFAP3) gene increases survival in sporadic amyotrophic lateral sclerosis. Proc Natl Acad Sci U S A 2009, 106:9004-9009.

41. Bolger TA, Zhao X, Cohen TJ, Tsai CC, Yao TP: The neurodegenerative disease protein ataxin-1 antagonizes the neuronal survival function of myocyte enhancer factor-2. J Biol Chem 2007, 282:29186-29192.

42. Blaeser F, Ho N, Prywes R, Chatila TA: $\mathrm{Ca}(2+)$-dependent gene expression mediated by MEF2 transcription factors. J Biol Chem 2000, 275:197-209.

43. Racioppi L, Means AR: Calcium/calmodulin-dependent kinase IV in immune and inflammatory responses: novel routes for an ancient traveller. Trends Immunol 2008, 29:600-607.

44. Ohmae S, Takemoto-Kimura S, Okamura M, Adachi-Morishima A, Nonaka M, Fuse T, Kida S, Tanji M, Furuyashiki T, Arakawa Y, et al: Molecular identification and characterization of a family of kinases with homology to Ca2+/calmodulin-dependent protein kinases I/IV. J Biol Chem 2006, 281:20427-20439.

45. Newton-Cheh C, Guo CY, Wang TJ, O'Donnell CJ, Levy D, Larson MG Genome-wide association study of electrocardiographic and heart rate variability traits: the Framingham Heart Study. BMC Med Genet 2007, 8 (Suppl 1):S7.

46. Thayer JF, Yamamoto SS, Brosschot JF: The relationship of autonomic imbalance, heart rate variability and cardiovascular disease risk factors. Int J Cardiol 2010, 141:122-131.

47. Sebastiani P, Solovieff N, Dewan AT, Walsh KM, Puca A, Hartley SW, Melista E, Andersen S, Dworkis DA, Wilk JB, et al: Genetic signatures of exceptional longevity in humans. PLoS One 2012, 7:e29848.

48. Puca AA, Daly MJ, Brewster SJ, Matise TC, Barrett J, Shea-Drinkwater M, Kang $\mathrm{S}$, Joyce $\mathrm{E}$, Nicoli J, Benson $\mathrm{E}$, et al: A genome-wide scan for linkage to human exceptional longevity identifies a locus on chromosome 4. Proc Natl Acad Sci U S A 2001, 98:10505-10508.

49. Reed T, Dick DM, Uniacke SK, Foroud T, Nichols WC: Genome-wide scan for a healthy aging phenotype provides support for a locus near D4S1564 promoting healthy aging. J Gerontol A Biol Sci Med Sci 2004, 59:227-232.

50. Beekman M, Blauw GJ, Houwing-Duistermaat JJ, Brandt BW, Westendorp RG, Slagboom PE: Chromosome 4q25, microsomal transfer protein gene, and human longevity: novel data and a meta-analysis of association studies. $J$ Gerontol A Biol Sci Med Sci 2006, 61:355-362.

51. Boyden SE, Kunkel LM: High-density genomewide linkage analysis of exceptional human longevity identifies multiple novel loci. PLOS One 2010, 5:e12432.

52. Geesaman BJ, Benson E, Brewster SJ, Kunkel LM, Blanche H, Thomas G, Perls TT, Daly MJ, Puca AA: Haplotype-based identification of a microsomal transfer protein marker associated with the human lifespan. Proc Natl Acad Sci U S A 2003, 100:14115-14120.

53. Nebel A, Croucher PJ, Stiegeler R, Nikolaus S, Krawczak M, Schreiber S: No association between microsomal triglyceride transfer protein (MTP) haplotype and longevity in humans. Proc Natl Acad Sci U S A 2005, 102:7906-7909.

54. Matsuzaka T, Shimano H, Yahagi N, Kato T, Atsumi A, Yamamoto T, Inoue N, Ishikawa M, Okada S, Ishigaki N, et al: Crucial role of a long-chain fatty acid elongase, Elovl6, in obesity-induced insulin resistance. Nat Med 2007, 13:1193-1202.

55. Morcillo S, Martin-Nunez GM, Rojo-Martinez G, Almaraz MC, Garcia-Escobar E, Mansego ML, de Marco G, Chaves FJ, Soriguer F: ELOVL6 genetic variation is related to insulin sensitivity: a new candidate gene in energy metabolism. PLoS One 2011, 6:e21198.

56. Cao H, Gerhold K, Mayers JR, Wiest MM, Watkins SM, Hotamisligil GS: Identification of a lipokine, a lipid hormone linking adipose tissue to systemic metabolism. Cell 2008, 134:933-944.

57. Shmookler Reis RJ, Xu L, Lee H, Chae M, Thaden JJ, Bharill P, Tazearslan C, Siegel E, Alla R, Zimniak P, Ayyadevara S: Modulation of lipid biosynthesis contributes to stress resistance and longevity of $C$. elegans mutants. Aging (Albany NY) 2011, 3:125-147.

58. Friedman N, Geiger D, Goldszmidt M: Bayesian Network Classifiers. Mach Learn 1997, 29:131-163.
59. Breiman L, Friedman JH, Olshen RA, Stone CJ: Classification and regression trees. Monterey: Wadsworth \& Brooks/Cole Advanced Books \& Software; 1984.

60. Crammer $K$, Singer $Y$ : On the algorithmic implementation of multiclass kernel-based vector machines. J Mach Learn Res 2001, 2:265-292.

doi:10.1186/1742-4933-9-7

Cite this article as: Ferrario et al.: The application of genetics approaches to the study of exceptional longevity in humans: potential and limitations. Immunity \& Ageing 2012 9:7.

\section{Submit your next manuscript to BioMed Central and take full advantage of:}

- Convenient online submission

- Thorough peer review

- No space constraints or color figure charges

- Immediate publication on acceptance

- Inclusion in PubMed, CAS, Scopus and Google Scholar

- Research which is freely available for redistribution

Submit your manuscript at www.biomedcentral.com/submit
C Biomed Central 